\title{
Model Test on a Metro Station Deep Foundation Construction Adjacent to Some Underground Structures
}

\author{
$\mathrm{Ke} W U^{1,2, a}$ \\ ${ }^{1}$ School of Civil Engineering, Beijing Jiaotong University, Beijing, 100044, China \\ ${ }^{2}$ Shenzhen Metro CO., LTD, Shenzhen, 518100, China \\ awk4223@163.com
}

Keywords: Metro Station, Adjacent to Underground Structures, Model Test, Deep Foundation.

\begin{abstract}
During the metro station construction, it is always inevitably encounter to lots of crossing some adjacent structures by side of those. The Chegongmiao subway station is adjacent to the first subway of Shenzhen and Fengshengding shopping street, which is on the planning construction of Shenzhen 11th subway. Based on the model test method, the effect of the deep foundation on the adjacent underground structures is investigated to efficiently simulate. The engineering measures to reduce the influence of construction are supported for determine the construction plan.
\end{abstract}

\section{Introduction}

The 11th subway line of Shenzhen is the first fast line which is taking into account the airport. The 11 th subway connects the Futian midcontinental region, the gulf midcontinental region with Shenzhen airport, which is one important traffic corridor. The Chegongmiao station stands the crossing of South of Shenzhen street and Xiangmi Lake Street. The type of station structure is the island station platform and 12 meters underground 2. It transfers the existing first line with the planning 7th, 9th lines. The exterior-protected construction of Chegongmiao station is adjacent to two underground structures, which are Fengshengding Shopping Street and one subway station in the first subway line. Therefore, it is one key of important facts that how to reduce the defective effect and control the lateral deformation of station during the foundation building. In order to guarantee the subway station to construct safety and control the deformation of the two underground structures in the permissible dimension, the building of new subway station is simulated based on model test [1-3].

\section{Model Test}

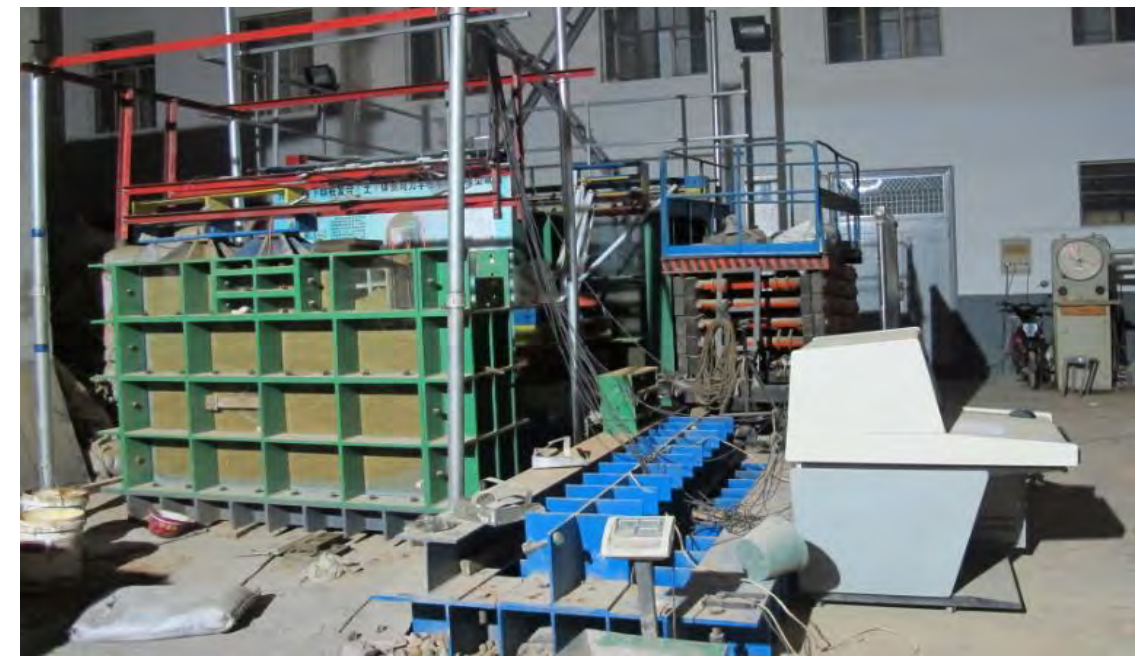

Fig. 1 Model Test

Based on the similar material test, the clay soil, sand and paraffin are combined one material according to a certain proportion. And the test device is researched and developed for analysis of subway station is 
shown in figure 1. In order to eliminate far-away boundary effect, the soil within 5 times of radium of foundation in wide and 6 times of height of foundation in depth is included into the computational region. The multi-point displacement meters are installed to investigate the deformation tendency of the adjacent underground structures as shown in figure 2[4-5]. When the deep foundation is excavated by open building construction, the surface soil is first excavated and the deep foundation is excavated through three steps. Any excavated steps has been becoming since the deformation of adjacent constructions is instability [6].

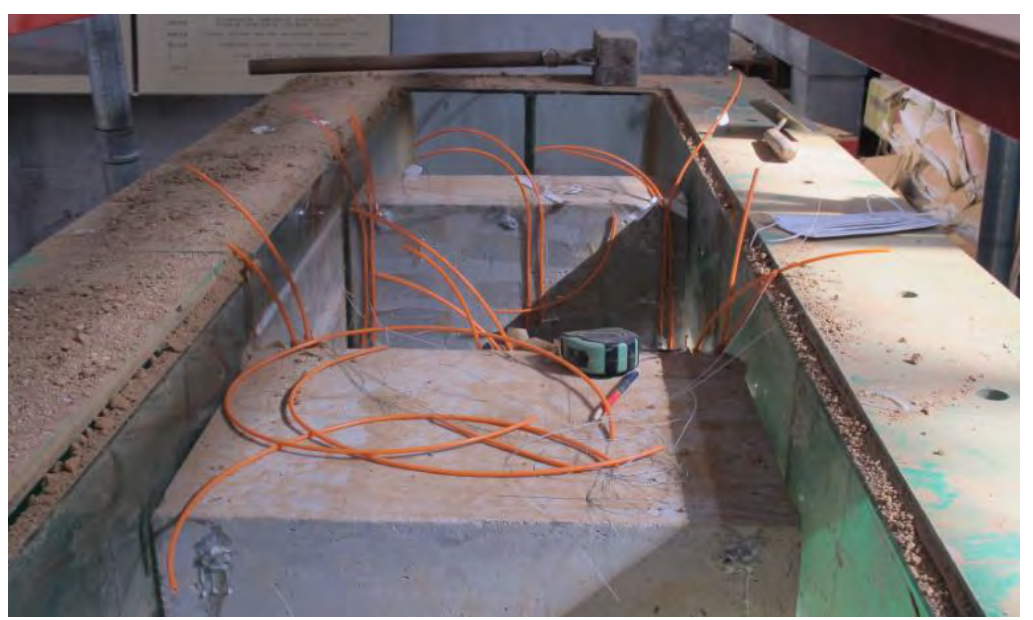

Fig. 2 Multi-point Displacement Meters

\section{Deformation Analysis}

Figure 4 and figure 5 are showing the horizontal deformation of the adjacent structures, the 1st line station and FengShengDing underground shopping street, by open excavation step by step. In the figures, the horizontal coordinate is deformation of side wall on the adjacent underground construction. It can be observed that: (1) the deformation curves for side wall of adjacent structures are basic similar. The top and bottom deformations of side wall for adjacent structures are smaller, but those of centre portion are larger. At the same time, the deformation curves are existing three inflection points, which are caused by three horizontal steel supports. The maximum deformations are all locating the 11 meters underground. (2) The horizontal deformation of Fengshengding structure is smaller than that of the 1 th subway station structure. The maximum horizontal deformations of Fengshengding and 1 th subway station structures by open excavation are 11 millimeters and 13 millimeters respectively.

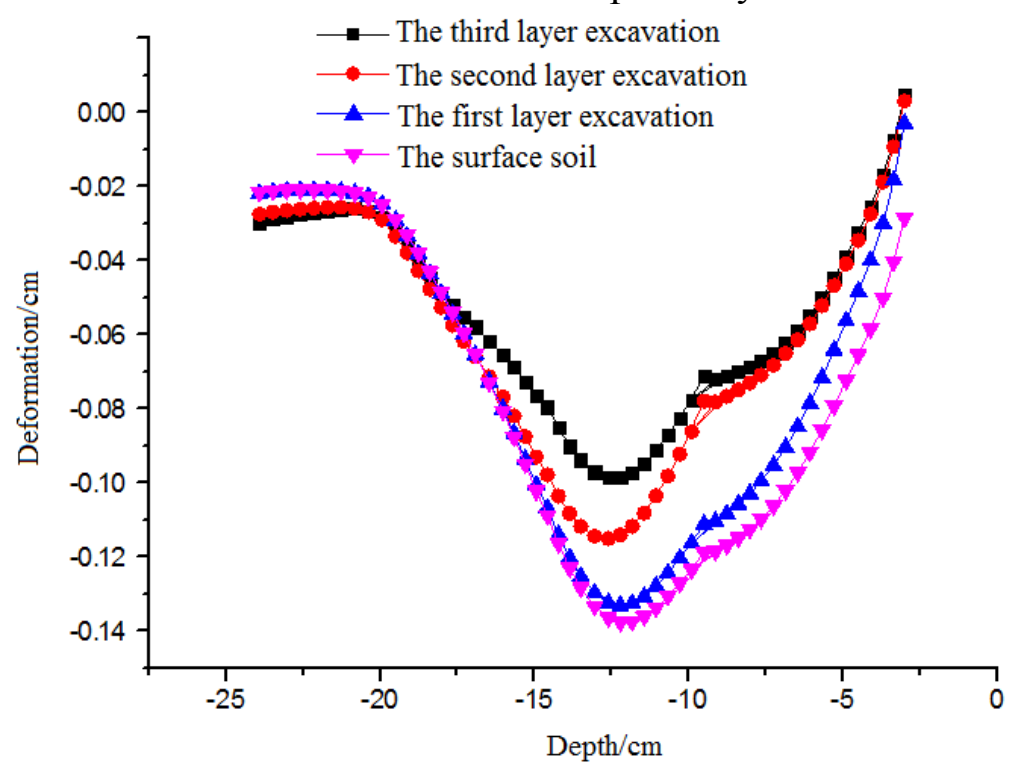

Fig. 3 The Horizontal Deformation of the 1st Line Station Structure During the Deep Foundation Construction 


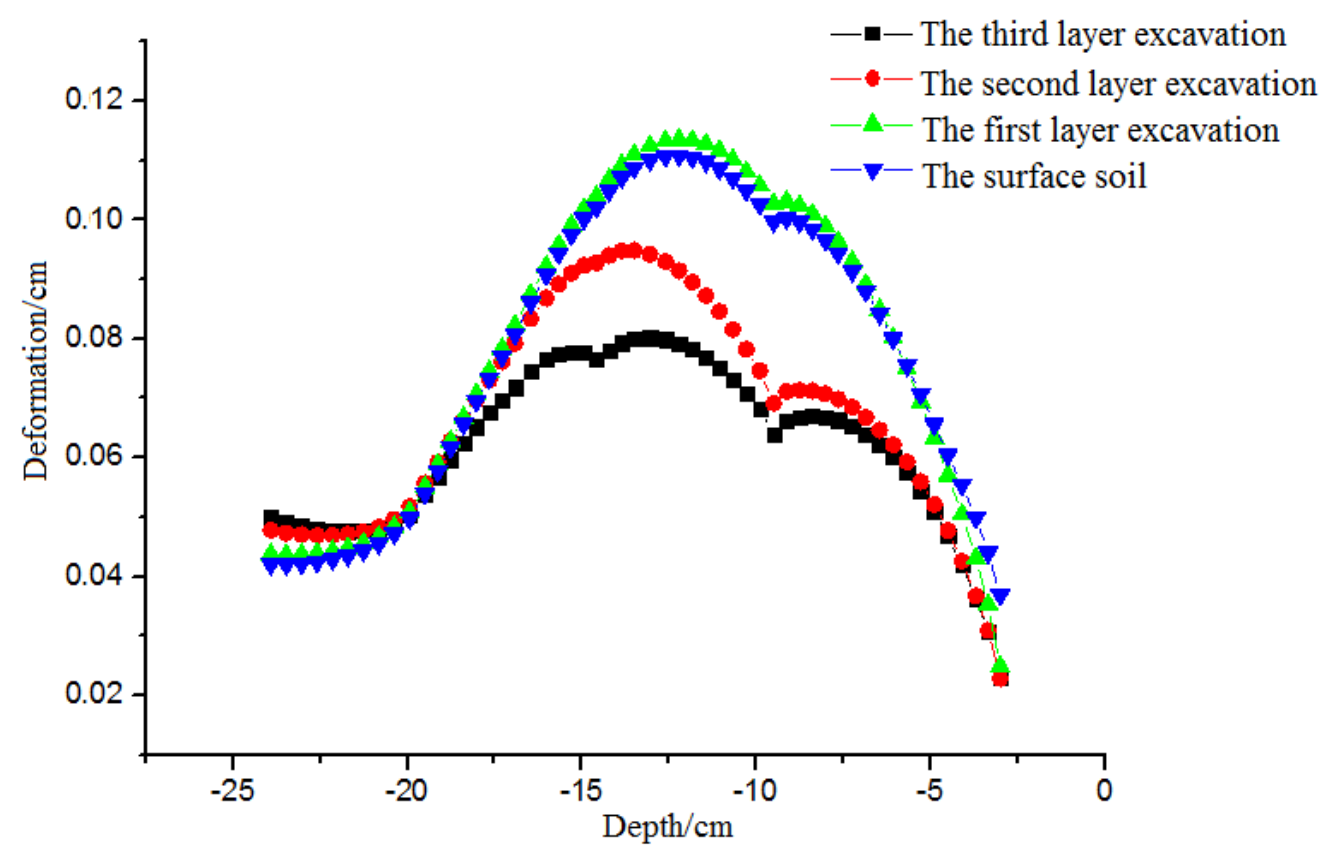

Fig. 4 The Horizontal Deformation of the FengShengDing Underground Shopping Street During the Deep Doundation Construction

\section{Deformation Control Measures}

According to the model test analysis, the adjacent underground structures are leaning to the deep foundation during the excavation. However, the horizontal support is easing the deformation tendency. So, it can be founded that: (1) the displacement monitoring of adjacent buildings should be strictly implied in order to early warning for constructors and guarantee the operation of adjacent structures. (2) The open excavation is safety, but it is influencing the deformation of structures greatly and widely. So, the horizontal support should be strictly carried out and measure the stress of those. And, the support stress is adjusted anytime to ensure the builder's life and the adjacent structures' safety.

\section{Concluding Remarks}

Based on the model test analysis, a reasonable simulation can be established to investigate the deformation of the adjacent structures by open excavation. It can be observed that: (1) the displacement monitoring of adjacent buildings should be strictly implied in order to early warning for constructors and guarantee the operation of adjacent structures. (2) The open excavation is safety, but it is influencing the deformation of structures greatly and widely. So, the horizontal support should be strictly carried out and measure the stress of those.

Since the accuracy of model test analyses may be affected by the model test size, a certain man-made errors probably exist in judgment of deformation state. Nevertheless these errors will be allowable in engineering practice.

\section{Acknowledgement}

This work was financially supported by the National Natural Science Fund (51008015).

\section{References}

[1]Ding Pengfei. Calculation and analysis on subway underground excavation station during construction stage [J]. Journal of North Traffic, 2010, 12: 56-58. 
[2]Chen Hong, Hu Jianguo. Analysis on architectural design method for mining metro station[J]. Chinese Journal of Underground Space and Engineering, 2010, 6(5):1001-1008.

[3]Jin Lei, Peng Jian, Liu Kun, Peng Fangle. Sorting theory and investigation of urban metro station[J]. Chinese Journal of Underground Space and Engineering, 2010, 6(1):1339-1342.

[4]Dai Kun. Comparative analysis on 3D computation model and 1D computation model for metro stations constructed by cut and cover method[J]. Tunnel Construction, 2010, 30(1):254-258.

[5]Liu Changyong, Gao Qiang. Analysis of top-down inverse construction method for a subway station [J]. Tunnel \& Underground Engineering, 2010, 28(1):95-98.

[6]Wang Wentong, Zhang Xiangduo. Analysis on monitoring results of open-cut metro stations reinforced by soil nails and anchoring cables[J]. Tunnel Construction, 2006, 26(1):14-17. 